\title{
Service Management: From Definition to Information Modeling
}

\author{
Constant GBAGUIDI, Simon ZNATY and Jean-Pierre HUBAUX \\ Swiss Federal Institute of Technology \\ TCOM Laboratory/ Telecommunications Services Group \\ CH- 1015 Lausanne- Switzerland \\ Phone: +412169352 59 Fax: +41216932683 \\ E-mail: \{gbaguidi, znaty, hubaux $@ @$ tcom.epfl.ch
}

\begin{abstract}
In this paper, we split the service management layer, as defined in the Telecommunication Management Network (TMN), into two sublayers, namely the service application management sublayer and the service network management sublayer. The former deals with managing the mapping of the non functional requirements into functional constraints over the telecommunication service of interest, while the second reveals the management details related to meeting the functional requirements over that service. At each sublayer, we propose an information model and emphasize the correspondence with the other sublayer.
\end{abstract}

\section{INTRODUCTION}

In the coming years, the number of stakeholders to be involved in service provisioning will increase because of the deregulation trend now taking over in the telecommunications market especially in Europe. According to the EURESCOM P.103 project [P.103], seven stakeholders should be considered, namely the service creator, the service provider, the service subscriber, the management provider, the resource provider, the network operator and the service user The former situation wherein roles among those actors were totally fuzzy, as services were the exclusive concern of the network operator, is turning over all around the developed world. To make the replacement situation attractive and fully promise-keeping, it is of high necessity first to make a separation of concerns between the network and its supported services, and afterwards to studiously tackle service creation, validation and management with respect to the roles played by the stakeholders.

Two fundamental attempts have been done to separate service related considerations from those of the network. First, the management community has defined a management framework known as TMN (Telecommunication Management Network) [M.3010] composed of five layers, namely the Business Management Layer (BML), the Service Management Layer (SML), the Network Management Layer (NML), the Element Management Layer (EML) and the Network Element Layer (NEL). Those layers are organized from the highest level of abstraction to the very lowest which is the network element view. Every layer basically provides some management service to the upper. The two upmost layers are concerned with service creation, validation and management, and the specification of the roles taken by the service stakeholders. The remainder of the framework deals with network management issues and mostly involves the network operator. Anyway, the TMN does not fill in the different layers and up to date only layers relating to network management are properly covered.

Second, the IN (Intelligent Network), aiming at a separation between networking and service issues, introduces a conceptual model composed of four functional planes, namely the service plane, the global functional plane, the distributed functional plane and the physical plane [Q.1201]. Every IN service is functionally split among those planes. So far, IN is the first standard which represents a service as a set of interactions among software components linked for execution by the service specific logic. However, subsequently to its functional approach, the IN conceptual model does not embed advanced distribution concepts, such as the object-based concepts advocated by the Reference Model of Open Distributed Processing (RM-ODP) [X.901]. Promoting distributed processing is motivated by the intrinsically spread nature of telecommunication systems which fits with a decentralized control and management scheme.

Efficient processing distribution is facilitated by the use of object-oriented approaches, since objects exhibit appropriate properties such as encapsulation (or data hiding, whereby users are prevented from directly manipulating objects) and autonomy whereby objects are responsible of managing their own behaviour [Herb94]. Therefore, as distribution is currently intensively promoted, the trend is towards the integration of the IN and RM-ODP concepts to the TMN layered architecture in the aim of managing telecommunication services. An early result is the Telecommunication Information Networking Architecture (TINA) [Dup94] delivered by the TINA-C ${ }^{1}$ consortium. Although IN concepts (which are service oriented) have been used in that architecture, the issues related to service management have not yet been completely tackled by TINA-C.

By contrast, service creation and validation have been somehow investigated, resulting in the use of methodologies borrowed from the software engineering area. In the future, the major shift in comparison with the current process for creating services will be the consideration of distribution concerns, thereby taking into account the possibility of having several management domains, especially those related to the service stakeholders. Moreover, skills gained through the development of IN services, such as the Service Creation Environment (SCE), might be helpful in implementing new services over the network. On the other hand, tools to be used for managing operating services are not yet clearly grasped.

This paper is intended to be a contribution to the service management work item by investigating a generic modelling of telecommunication services. Such a modelling emphasizes the generic service features and structures the related information conventionally using an object-oriented approach, namely the Object Modeling Technique (OMT) [Rumb91] for the purposes of this document. The used approach enables the distribution of services to be "straightforward" from the modelling of the latter. To this end, this

1. Information about TINA-C is available from http:// www.tinac.com/. The TINA-C outcomes have been somehow enhanced by the EURESCOM P.103 project. 
paper is organized as follows. Section 2 concentrates on clarifying the service related terminology, especially service management. In Section 3, a generic information model of telecommunication services is proposed, the usage of which is illustrated in Section 4. Through that usage statement, we aim at defining the service management layer in a clear and graphical manner by taking a videoconferencing example.

\section{THE SERVICE CONCEPT AND THE RELATED TERMI- NOLOGY}

Service can generically be defined as a collection of capabilities, with each capability being a set of actions performed by an entity (acting like a server) in response to a (constrained) request issued by another entity (being the client). Among actions to be taken by the server, some may need interactions with other entities as illustrated in Figure 1, where (N+1)Capability 2 calls for performing (N)-Capability3 and (N-1)Capability 1 . Hence, the service capability concept is recursive.

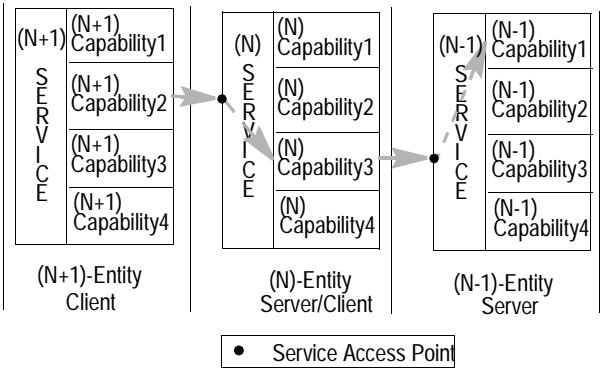

Figure 1: The service concept as related to a client/server model.

The given service definition points to several considerations. First, to be accessed a service must provide access points (where the service could be requested) and interfaces (declaration of the supported service capabilities). Second, some authentication mechanisms should be performed in order to preclude intruders from accessing the service offered by the entity of interest. Third, talking of constrained requests means the possibility of putting some requirements over the service capabilities. Therefore, the service concept is basically associated with the provision of access points, authentication procedures, and techniques for meeting the clients requirements.

In the telecommunication market, services are divided into two categories, which are bearer services and teleservices [I.210]. The former category is concerned with providing the capability for information transfer between two network access points, whilst the second kind of services deals with supplying the full capacity for communication thanks to functions provided by dedicated centers, which may be service specific systems (like the so-called third-party equipments), user terminals or networks.

Teleservices are of two kinds: basic teleservices and value-added teleservices. This document preferably focuses on the former which are collections of communication facilities offered to the users. Examples are multicasting, multiplexing, telephony, etc. Value-added teleservices aggregate basic teleservices and supplementary services which are intended to modify and supplement the former by supplying some comfort to the user. Call screening and call forwarding are examples of supplementary services. Note that IN essentially addresses the last kind of services.

At the user site, the provision of a teleservice is enabled by the service access tools, which are terminal components, especially software or service specific boards. For instance, among tools that the users need for accessing MBone ${ }^{1}$ [Mac94] are $n v$ (net video), vat (visual audio tool) and $w b$ (whiteboard). As a teleservice, MBone also introduces specific routers called mrouters (service specific equipments). At last, applications are defined as the usages made of a teleservice. A summary of the MBone example illustrating the main terms introduced above is given in Figure 2.

\begin{tabular}{l|l|l|}
\cline { 2 - 3 } Applications & \multicolumn{2}{|c|}{$\begin{array}{c}\text { videoconferencing, } \\
\text { teleteaching, etc. }\end{array}$} \\
\cline { 2 - 3 } Service access tools & $\begin{array}{c}\text { Software } \\
n v, \text { vat,wb }\end{array}$ & $\begin{array}{c}\text { Equipments } \\
\text { mrouters }\end{array}$ \\
\cline { 2 - 3 } Teleservice & $\begin{array}{c}\text { Basic } \\
\text { MBone }\end{array}$ & $\begin{array}{l}\text { Supplementary } \\
\text { Services }\end{array}$ \\
\cline { 2 - 3 } Bearer service & \multicolumn{2}{|c|}{$I P$ class $D$} \\
\cline { 2 - 2 } \multicolumn{1}{c|}{ Figure 2: } & Illustrating the service related
\end{tabular}

The choice of the equipments and service access tools depicted in Figure 2 is motivated by the requirements over the service of concern. Therefore, teleservices can be described by the requirements they are subject to and the used equipments, service specific systems and networks as well (Figure 3). Two kinds of requirements are generally outlined [Str95 and SoA92]. First, the non functional requirements cover external and observable characteristics of the implemented service, i.e., the service as perceived by the service stakeholders, especially the users, at operation time. Examples of non functional requirements are the users QoS expectations. Second, functional requirements address the internal composition and deployment of the service of interest, including the network environment of the latter. Examples of functional requirements are exigencies related to feature interactions among the service components.

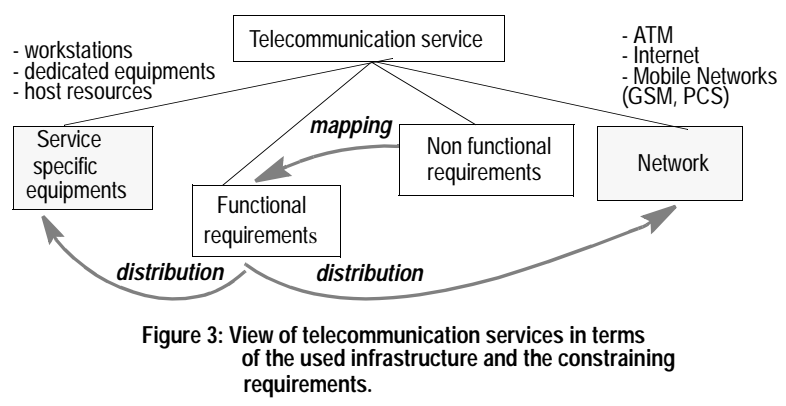

The trial along the service life-cycle is the mapping of the stakeholders non functional requirements (external perception) into functional requirements (internal deployment) and the distribution of the latter over the infrastructure used for the purposes of the service. At service creation time, that mapping leads to the choice of the service specific equipments and, possibly, to commercial agreements with some network operators. After deploying the service, that mapping is achieved by a functionality called service management. Therefore, the latter can be defined as the intelligence, the set of mechanisms to be taken at operation time both for mapping the service constraining requirements over the used infrastructure, and for managing that mapping. Those requirements are captured from the seven

1. MBone is a teleservice providing the users with the multicasting facility offered by the class D addressing scheme of the IP (Internet Protocol) [RFC1112 and Com91]. 
service stakeholders outlined in [P.103]. When only the users non functional requirements are considered, service management is restricted to QoS management, which naturally is a crucial part of the former.

This Section states the terminology which underlies the substance of this paper. In the following, we propose a generic information model of telecommunications services. As extensions to teleservices, supplementary services are not specifically addressed in the ensuing Section. Focus is rather on the former services which are more complex to design, as they involve a lot of resources distributed over several management domains.

\section{A GENERIC INFORMATION MODEL OF TELECOMMU- NICATIONS SERVICES}

We characterize telecommunication services by two main features, which are their general aspects and their dynamics (Figure 4). The general aspects basically describe services regardless of their operation as to provide communication facilities to the users. On the other hand, the service dynamics integrates the behaviour and description of services when these are used for communication purposes. Each of those features is examined in turn below.

\subsection{General Aspects}

Basically, a teleservice is made up of supplementary services, a supporting infrastructure, a number of policies, functions, especially management functions, and access points (Figure 4). The supporting infrastructure consists of provided systems and networks. Networks are either supplied by the service provider or made available by network operators by virtue of commercial agreements. The service policies include security, accounting and billing policies. Policies relating to agreements between the service provider and other parties such as the network operators are also considered. Relying on [GZH95], among functions needed for managing services are QoS negotiation, QoS monitoring and control, and session management. Concerning access points, they provide gates to the service. Any user connecting to the service via an access point induces the creation of a user access session. This creation is the "guarantee" for the calling user to be provided later on with all service capabilities or a subset of these.

\subsection{Service Dynamics}

At operation time, the service dynamics is described by its behaviour and the on-going communication groups. The service behaviour, depicted by the ServiceEntity class in Figure 4, records statistics about the service utilization and performance. Moreover, each communication group involves some systems and networks. These are not necessarily those supplied by the service provider or made available thanks to existing commercial agreements (see above description of the general aspects). Systems and networks of interest here may be provided respectively by the service user (e.g., workstations) and network operators not committed to the service provider anyhow. The information models of systems and networks are not emphasized here. Many generic network information models have been proposed, especially in [M.3100], [TINA93], [Zna93] and [Gasp95]. With regard to systems, a simplified representation is depicted in Figure 4, highlighting the access points to their provided service, the system components such as devices, and the user sessions being held within the system. The term "user" here generically means a single user or a group of users. Within a user session there are a description of the user's profile (UserAgent), the applications launched in the objective of meeting the user's requirements, and the topology of the session. The topology is an abstraction of the system resources being consumed by the user's applications. As topologies embed the same elements as Logical Connection Graphs (LCG) [Moy93 and P.103], they are described below when characterizing the latter. The differences between LCGs and user session topologies will also be emphasized.

Regarding the applications running within a user session, they are considered together with their QoS constraints and the statistical metrics about their behaviour (ApplicationEntity), e.g., information related to their bugs and the latter's occurrences.

The last element to be described within a communication group (Figure 4) is the service session, i.e., the set of activities carried out among service specific systems and networks over some temporal period, this period being the time frame of the interactions among the users participating in the session. We do not think that a session should be defined as in [P.103], where it is assimilated to the temporal period during which some activities take place among the service users. The most relevant feature of a session is less its duration than the activities to be achieved.

A service session is composed of an entity, which gathers statistical metrics about the session, session policies and Logical Connection Graphs (LCG). Service session policies span voting policies and consistency policies [SWS95]. The voting policies state how decisions about the session are made. Two kinds of voting policies can be distinguished: implicit voting and explicit voting. The former does not specifically require a voting procedure. For example, a videoconferencing system may automatically select the current speaker's image and voice (the current active side) to be multicast to the other partners in the session, instead of multicasting the information originating at all of endparties. In contrast to the first kind of voting policies, the explicit voting requires decisions to be made by a subset of session members.

Consistency policies state the causal holding of the events occurring at the end-parties. Since applications like videoconferencing are controlled in a distributed way, each site has its own view of the global session. Therefore, for the user side session to be eventually consistent, the restitution of all events should be guaranteed versus the ordering of their occurrence, even if the temporal intervals between those events are not stringently kept. A broader description of consistency policies can be found in [SWS95].

Managing a service provisioning system at runtime necessitates a logical representation of the activities being carried by that system, especially service sessions (Figure 4). For this purpose, LCGs provide a powerful means for abstracting real communications and highlight the resources involved in a session in progress. Resources referred to are those provided in the end of supplying the whole service, i.e., both network and system (e.g., workstation or third-party equipment) resources. As the network management parameters are well-known within the management community, the challenge will now be to point out features which are relevant for managing service devoted (or specific) systems. An early result can be found in [GZHV96], which describes system resources with the proposal of a generic information model furthermore applied to MPEG-2 video codecs. 

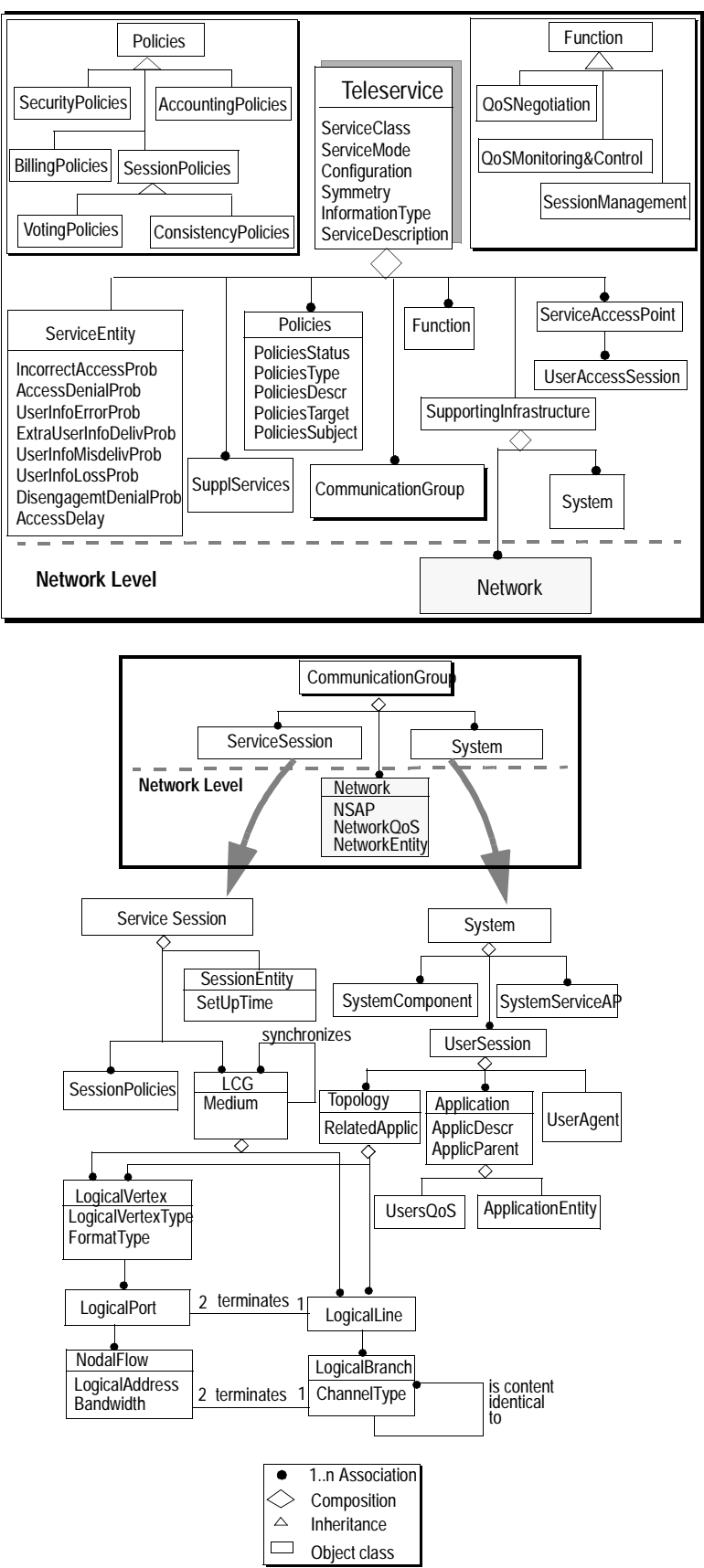

ServiceClass: \{best-effort, guaranteed, compulsory\} ServiceMode: \{connection mode, connectionless mode) Configuration: \{point-to-point, etc.\} Symmetry: \{unidirectional, bidirectional\} InformationType: \{audio, video, text, graphics, picture)

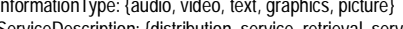
ServiceDescription: \{distribution_service, retrieval IncorrectAccessProb: incorrect access probability AccessDenialProbability: access denial probability UserlnfoErrorProb: error probability for the user information
ExtraUserlnfoDelivProb: reliability for extra user info. delivery UxtraUserInfoDelivProb: reliability for extra user info. delivery UserInfoLossProb: user information loss probability DisengagemtDenialProbability: disengagement denial prob. AccessDelay: service access delay

LogicalVertextype: \{signal_converter, end_vertex, etc.\} FormatType: \{ MPEG1, MPEG2, M-JPEG, etc.\} ChannelType: \{ISDN, etc.\}
PoliciesStatus: \{appended, activated, PoliciesType: policies type PoliciesDescr: policies description PoliciesTarget: target of the policies PoliciesSubject: subject of the policies NetworkQOS: QoS requested from the NetworkQoS: QoS request
network

NetworkEntity: network behaviour Medium: processed information type RelatedApplic: related application ApplicDescr: application description ApplicParent: application parent LogicalAddress: flow logical address Bandwidth: quantity of information conveyed by the nodal flow SetUpTime: session setup time

Figure 4: A generic information model of telecommunications services.

Figure 5 introduces an LCG in the framework of a videoconferencing session. Streams generated by the three sources involved in the session are multiplexed, and the resulting stream is multicast back to the originating vertices. Resources involved at end-systems are highlighted, and their features (especially coding schemes) alike. The incompatibility among those features leads to the introduction of signal converters between the information generators and the resources used for multicasting purposes.

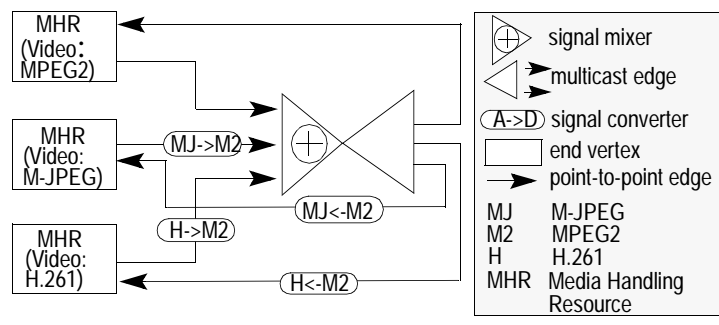

Figure 5: Abstracting a real communication using an LCG.

Every LCG relates to a specific medium, as proposed in [GZH95], in order to separate the processing of the media involved in a service session. Doing so helps deal with situations wherein some session end-parties are not able to process information related to certain media. For instance, in a videoconferencing session, a user whose equipment has no capabilities for processing video information should be able to receive the single audio component and participate in the session somehow. Therefore, having one LCG per medium permits dealing with capability mismatching among end-systems.

A conceptful representation of LCGs is given in Figure 6. These are composed of logical vertices and logical lines. Logical vertices basically represent the resources involved in the session, and logical lines the connections among the ports exhibited by the resources. The logical ports associated with logical vertices aggregate nodal flows which are connected by logical branches. The notion of logical flow as depicted in Figure 6 is represented by the relationship "is content identical to" between logical branches in Figure 4.

It is worth noting that LCGs always feature end logical vertices, such that there is no branch going out of them. By contrast, session topologies mentioned earlier in this subsection when describing service specific systems do not necessarily exhibit that "self-containment" property. For instance, in Figure 5 , signal converters may be provided by a third-party equipment (system), thus leading to a topology consisting in a single vertex supplying two nodal flows. That makes the difference between the concepts of LCG and session topology.

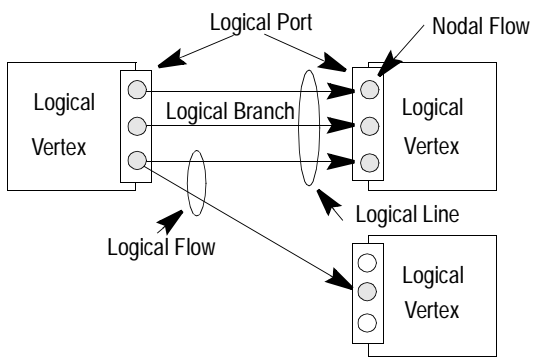

Figure 6: Representing an LCG with the associated concepts.

Like any information model, the one proposed in this Section may be perceived too much abstract to be successfully used for the management of concrete services. The following Section states the utilization of this model by considering the videoconferencing session depicted in Figure 5. 


\section{USAGE STATEMENT OF THE DESIGNED MODEL}

The goal of this Section is twofold. It is intended, first, to clarify the use of the designed model in connection with the service management layer, and, second, to relate this work to other ones, especially [X.901] and [CASSIOPEIA95].

The service provisioning and management layer can be divided into two sublayers, namely the service application and the service network management sublayers (see Figure 7). The former is concerned with managing the user's perception of the service, i.e., the service application, which is videoconferencing for our purposes. At this sublayer, objects to be managed are the application level flows exchanged among the users and the global environment which performs the requested application. Application flows are characterized by their direction (origin and destination), their QoS requirements and their related information type (audio, video, etc.). All activities achieved within the service application provisioning system as results of the interactions with a group of end-users constitute the application session. The modelling of the latter is shown in Figure 8 as an aggregation of session ports, user agents and the functions to be achieved for processing the users requests and flows carried away through the session ports. Those functions are supported by a processing network called service network. In Figure 7, this network is represented with respect to the LCG example in Figure 5. The SSE (Service Specific Equipment) on top of the packet network deals with multicasting issues, whilst the other ones perform signal conversion.

At this point, the information model proposed in Section 3 is targeted to be used at the service network management sublayer, which is the lowest sublayer contained by the service management layer. The service session is defined as the entity which controls both the correct implementation of the service policies and the mapping of the stakeholders non functional requirements into functional exigencies. In this respect, service sessions are responsible of carrying the interactions among the involved end-systems. It is worth noting that end-users visible at the service application management sublayer are mapped into end-systems at the service network management sublayer. The reason is the following. End-users "view" application flows in their native form, regardless of the processing activities carried out by the entire videoconferencing system. On the other hand, the service network considers the overall framework that provides the requested service application. Thus, this network features end-systems, networks and service dedicated equipments, each with its own constraints (e.g., format constraints, packet size, protocol, etc.) over the information to be processed.

Moreover, the service management sublayers are constructed at either step along the service life-cycle. With respect to [X.901] which states a paradigm for achieving distributed processing, we can relate the construction of the service application management layer to the enterprise viewpoint and part of the information and computational viewpoints (Figure 7). As a matter of fact, this sublayer is concerned with managing the user's access to the service of interest as well as the user's QoS expectations. Furthermore, the service network management sublayer essentially addresses the service deployment, which is related to the computational, engineering and technology viewpoints described in [X.901]. Note that the Open Services Architectural Framework $(\mathrm{OSA})^{1}$ [CASSIOPEIA95] introduces

1. The OSA framework has been carried out by the RACE II project R2049 CASSIOPEIA. concepts of service machine, which corresponds to the computational viewpoint, and service network which is mapped onto the engineering and technology viewpoints.

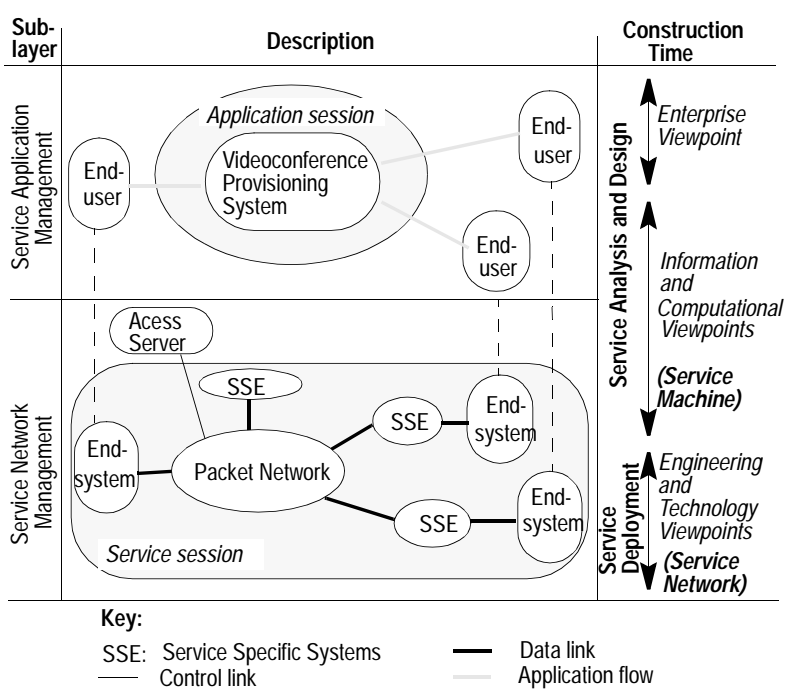

Figure 7: A layered description of service management.

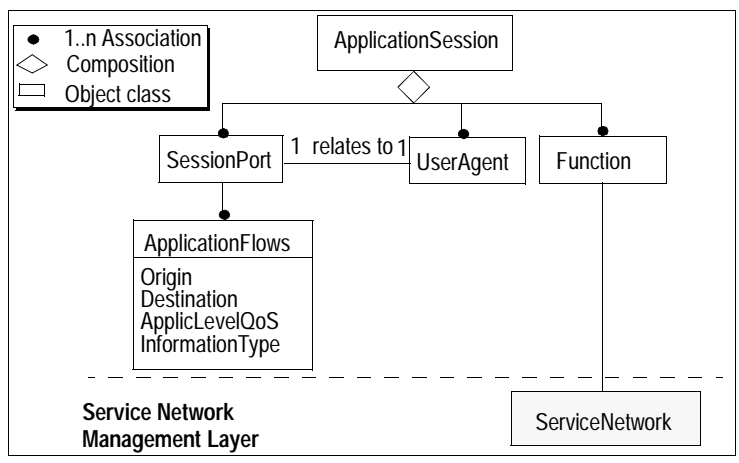

Figure 8: A generic modelling of application sessions.

In this Section, we have achieved the description of the service management layer by splitting this one into two sublayers, namely the service application management sublayer and the service network management sublayer. In connection with the service description given in Section 2 in terms of the employed infrastructure and the constraining requirements, the service application management sublayer deals with managing the mapping of the non functional requirements into functional constraints over the deployed service. The service network management sublayer is rather concerned with the details related to meeting the functional requirements over services. We have also investigated the construction of the aforementioned sublayers with respect to the RM-ODP [X.901]. This investigation led us to relate the construction of the service application management sublayer to the enterprise and part of the information and computational viewpoints, while the construction of the service network management sublayer fits on the engineering and technology viewpoints.

\section{CONCLUSION}

The investigation of a generic modelling of telecommunication services led us essentially to three achievements. First, the fuzziness around the service concept led us to state more 
precisely the service related terminology taking into account what is considered as the future of the telecommunication market, namely deregulation inducing several service stakeholders. We especially define service management versus QoS management, the former being the intelligence to be held for meeting all of the requirements over a service at operation time, whilst QoS management restricts the former to meeting the users non functional expectations.

Second, through the proposed information model of telecommunication services, we highlighted the representation of sessions in the shape of LCGs. That model features both the information needed at the service operation time (e.g., communication sessions) and general information such as the service policies, equipments, the possible commercial agreements among the stakeholders, etc.

At last, stating the use of the proposed information model led us to consider the splitting of the service management layer into two sublayers, which are the service application and service network management sublayers. The first sublayer addresses the management of the service provisioning system, with the latter pictured as a "black box". The second sublayer reveals the equipments involved in the provision of the service of interest, picturing the latter as a "white box". The information model designed in Section 3 can be considered as a "white box" description of telecommunication services. The "black box" representation is provided by the concept of application session as modelled in Figure 8.

A future issue to this work is its application to the management of a real videoconferencing application, the implementation of which is underway over a distribution platform, namely OSF DCE (Open Software Foundation's Distributed Computing Environment) [Lock94]. That integration will hold in a project called OAMS (Open management Architecture for Multimedia Services over ATM) led within our laboratory.

\section{ACKNOWLEDGEMENTS}

The authors would like to acknowledge Prof. Noemie Simoni, from ENST, Paris, France, and Prof. Tuncay Saydam, from University of Delaware, Newark, Delaware, USA. The discussions the authors had with those personalities considerably contribute to writing this paper, especially the second Section.

\section{REFERENCES}

[CASSIOPEIA95] Project Cassiopeia, Open Services Architectural Framework for Integrated Service Engineering, Deliverable R2049/ FUB/SAR/DS/P/023/b1, Vers. 4, March 1995.

[Com91] D.E. Comer, Internetworking with TCP/IP, Vol. 1, Prentice Hall, Englewood Cliffs, New Jersey, 1991.

[Dup94] F. Dupuis, R. Minerva, J. Bloem, H. Hammainen, J. C. Moreno, The TINA-C Architecture as Related to IN and TMN, International Conference on Intelligence in Networks ICIN '94, October 94, Bordeaux, France.

[E.800] ITU-T Rec. E.800, Quality of Service and Dependability Vocabulary, 1993.

[Gasp95] J.-P. Gaspoz, Object Oriented Method and Architecture for Virtual Private Network Service Management, Ph.D. dissertation no. 1446 presented at TCOM/EPFL in November 1994, Lausanne, Switzerland.

[GZH95] C. Gbaguidi, S. Znaty, J.-P. Hubaux, A Generic Service Management Architecture for Multimedia Multipoint Communications, IFIP IN 95, August 1995, Copenhagen, Denmark.

[GZHV96] C. Gbaguidi, S. Znaty, J.-P. Hubaux, O. Verscheure, Management of Multimedia Resources: From a Generic Information
Model to its Application to an MPEG2 Video Codec, INDC '96, Trondheim, Norway, June 1996.

[Herb94] A. Herbert, Distributing Objects, Distributed Open Systems, 1994.

[I.140] ITU-T Rec. I.140, Attribute Technique for the Characterization of Telecommunication Services Supported by an ISDN and Network Capabilitites of an ISDN, March 1993.

[I.210] ITU-T Recommendation I.210, General Aspects of Services in ISDN, 1988.

[I.211] ITU-T Rec. I.211, B-ISDN Service Aspects, March 1993.

[I.350] ITU-T Rec. I.350, General Aspects of Quality of Service and Network Performance in Digital Networks, including ISDNs, March 1993.

[I.356] ITU-T, I.356, B-ISDN ATM Layer Cell Transfer Performance, November 1993.

[Lock94] H.W. Lockhart, Jr, OSF DCE : Guide to Developing Distributed Applications, McGraw-Hill, Inc., 1994.

[M.3010] ITU-T Rec. M.3010, Principles for a Telecommunication Management Network, October 1992.

[M.3100] ITU-T Rec. M.3100, Generic Network Information Model, October 1992.

[Mac94] M.R. Macedonia, D.P. Brutzman, MBone Provides Audio and Video Across the Internet, IEEE Computer, April 1994.

[Moy93] S.L. Moyer, D.S. Rouse, A Connection Manager for Flexible Specification and Transparent Location of Special Resources, GLOBECOM'93, pp. 1526-1530.

[P.103] T. Vaarnamo, Framework for Service Description with Supporting Architecture: Final, EURESCOM P103.TF.TV.75, Deliverable 6a, December 1994

[Q.1201] ITU-T Draft Rec. Q.1201, Principles of the Intelligent Network Architecture, September 1991.

[RFC1112] S. Deering, Host Extensions for IP Multicasting, IETF RFC 1112, August 1989.

[Rumb91] J. Rumbaugh, et al., Object-oriented Modeling and Design, Prentice Hall International Ed., 1991.

[Scla94] J. Sclavos, N. Simoni, S. Znaty, Information Model: From Abstraction to Application, IEEE NOMS'94, Florida, USA.

[SoA92] ICOM, GMD, Methods for QoS Verification and Protocol Verification in IBC-SoA Report, Report R088/ICO/DPD/DS/P/000/b3, Vers. 3, September 1992.

[Str95] L. Strick, J. Meinköhn, Enterprise Modelling for the Design of Telecommunication Management Systems, in Proc. of Conference TINA 95 on Integrated Telecommunications and Distributed Computing, pp359-369, Melbourne, Australia, February 1995.

[SWS95] S. Shenker, A. Weinrib, E. Schooler, Managing Shared Ephemeral Teleconferencing State : Policy and Mechanism, Internet-Draft registered as draft-ietf-mmusic-agree-00.ps, July 1995.

[TINA93] L. Richter, et al, Network Resource Information Model Specification, Document label TB_C2.LSR.001_1.0_93, TINA Consortium, December 1993.

[X.140] ITU-T Rec. X.140, General Quality of Service Parameters for Communication via Public Data Networks, September 1992.

[X.901] ITU-T Draft Rec. X.901 | ISO/IEC 10746-1 ODP Reference Model Part 1. Overview, July 1994.

[Zna93] S. Znaty, QoS of a Multi-network: From a Model to its Realization, Ph.D. Dissertation no. E020 presented at Telecom Paris, 1993, Paris, France. 